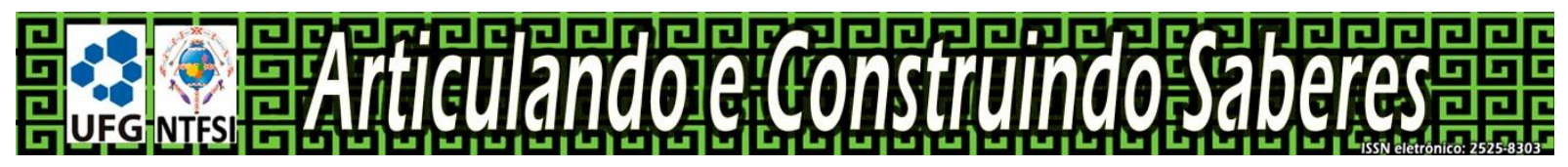

DOI: $10.5216 /$ racs.v4.59296

\title{
La planeacion desde el enfoque del metodo inductivo intercultural
}

Georgina Marcelo Hernández ${ }^{1}$

\section{RESUMEN}

Este texto trata de la planificación docente como actividad fundamental de la actividad del profesor. En esta dirección, busca pensar en una planificación que tenga en cuenta los saberes propios de la comunidad. Es en este contexto que se establecen principios y dinámicas de trabajo que apuntan a la formación y el aprendizaje para el bien vivir. Además, se describe cómo el proyecto Milpas Educativas actúa en la dirección de generar prácticas educativas contextualizadas en los saberes comunitarios que apuntan al bien vivir.

PALABRAS CLAVE: Bien vivir. Educación. Planificación.

\section{O planejamento desde o enfoque do Método Indutivo Intercultural}

\section{RESUMO}

Este texto trata do planejamento docente como atividade fundamental da atividade do professor. Nesta direção, busca se pensar em um planejamento que leve em consideração os saberes próprios da comunidade. É neste contexto que se estabelecem princípios e dinâmicas de trabalho que visam a formação e o aprendizado para o bem viver. Ademais, descreve-se como o projeto Milpas Educativas atua na direção de gerar práticas educativas contextualizadas nos saberes comunitários que visam o bem viver.

PALAVRAS-CHAVE: Bem viver. Educação. Planejamento.

Hablar de planeación nos lleva a varias incógnitas sobre todo a pensar ¿cómo hacerlo? ¿Qué hacer primero? ¿De dónde partir? ¿Qué elementos debo tener para partir? ¿Qué aspectos debo considerar? ¿Qué características debe prever mi planeación? ¿Cómo organizarlo? ¿Quiénes intervienen? ¿De qué forma puedo hacerlo? ¿Quién me puede ayudar? ¿Qué conocimientos debo

\footnotetext{
${ }^{1}$ Maestra do povo Náhuatl. Estado de Puebla, México. E-mail: gmarcelohernandez8@gmail.com.
} 
retomar? ¿qué tipo de aprendizaje desarrollar? ¿dónde parte el conocimiento? ¿Quién y donde se hace la actividad?

Son premisas que como docente siempre se pregunta uno, considerando que muchas de las veces se encierra uno en el grupo escolar o la escuela, y lo que siempre en el servicio se enfrenta uno como docente con muchas interrogantes debido a que en la escuela, por los tiempos, programas, actividades curriculares es la que ha hecho falta en hacer hincapié en la práctica y dar elementos sustantivos que lo permitan, ahora el desarrollo no está alejada de la teoría es necesario que como docente se tenga claro, firme, decidido, ¿qué se quiere trabajar? ¿Cuál es el objetivo? ¿a quién debe dirigirse el objetivo? ¿Qué tipo de objetivo debe plantearse? Considerando que el servicio docente aunque existe exclusivo para educación indígena muchas de las veces el objetivo no va acorde con el contexto al que se plantea, por lo tanto aunque existan buenos resultados memorísticos, lo que se obtiene es una ambivalencia y rechazo de su propio espacio del educando porque las actividades curriculares dan un mensaje distinto al previsto, imponiendo de manera indirecta lo urbano, por lo que el niño crece con la idea de que lo que existe en su espacio, a su alrededor son aspectos que no son dignos de considerarse dentro del entorno curricular, se requiere de una actitud positiva, hacia el propio servicio, hacia el espacio donde ha tocado estar.

$\mathrm{Si}$ es una comunidad donde aún se encuentra en una cultura inmersa con muchos elementos del legado cultural, es aceptar en que ese conocimiento implícito contiene mucha ciencia que aún no ha sido descubierta, que tiene valor social, natural, que ha venido de generación en generación, que se resiste a quedarse en los tiempos y en el olvido, por el simple hecho de ser efectivo, situado, en una interacción hombre naturaleza. En donde a pesar de la imposición continua moderna, el valor del sentido cosmogónico, aún prevalece, se empodera.

Otra de las incógnitas que se tiene cuando ya existen elementos teóricos sustanciales hace falta entender el enlazamiento, la vinculación de esa contextualización con el currículo escolar.

Uno de los problemas que se han encontrado del sistema educativo es que muchas veces se culpa al estado, a la Nación porque los grupos indígenas han estado olvidados en cuestión educativa, viéndolo desde otro ángulo de la óptica, no es por defender al sistema, sino que los mismos docentes que se ha formado en una educación occidental, en un programa curricular que ha permitido la descontextualización, el alejamiento de su propio espacio, creando ideas "mejores" o "mejores personas", donde se empodera lo material, alejando lo sentimental que va enlazado con la mente, cuerpo, corazón, en donde lo mejor es vivir bien, pero ese bien no es que 
el cuerpo, mente, corazón tenga salud, estabilidad emocional, sino que se mira como lo bien todo lo material que se pueda acercar como una vanidad de lujos, por lo tanto muchos de los que están en el servicio docente como salieron de una comunidad, de un pueblo, donde por hablar una lengua, por ser y formar parte de una cultura, de una identidad que es señalada en la calle, en el camino, en la sociedad urbana, niegan su propia identidad empapándose de lo que la sociedad externa le proporciona desde vestido, objetos distintos, por lo tanto al volver o trabajar en las comunidades a pesar de tener varios elementos que lo identifica, es un objeto de sometimiento y de imposición también por que es el que llega con las modas, con modales que ha aprendido en el exterior, entonces como salir de la propia cultura implica olvidarse de lo que en algún momento obtuvo, o en su defecto rechazando algunos elementos como símbolo de denigración, de discriminación, de rechazo propio.

Entonces se puede decir que a pesar de la existencia de un espacio que es para atender a las comunidades, un sistema con identidad e identitación, la formación docente es la que no ha quedado claro, es la que le hace falta encauzar para que pueda aceptarse primero así mismo, con todas las características que le han sido dadas desde el espacio de la cultura donde se ha encontrado inmerso, así como el reconocimiento propio de lo que hay, de lo que es, de donde viene, y el objetivo claro de ¿dónde va a realizar su servicio? ¿Cómo se le puede decir al niño que valore su cultura? Si desde los que están en el proceso de formación la desvaloran.

Por lo tanto se puede decir que el servicio no ha dado lo que le corresponde, o en su defecto no ha correspondido esa atención a la que le ha sido asignada, que tiene mucha influencia actitudinal de los que están en el servicio, ya que al aprobarse, firmar los convenios, los acuerdos como él 169 de la OIT, la UNESCO, los acuerdos de San Andrés promovidos por el EZLN en el año de 1996, que dieron bastantes elementos de empoderamiento, de aceptación, de lucha hacia esa atención, pero que no se ha encontrado el camino perfecto para poder conducirlo y ser aterrizado en su tiempo de manera clara, pertinente, también tenemos la constitución política en su artículo $2^{\circ}$.

Los derechos lingüísticos para la educación bilingüe, los lineamientos para una educación intercultural bilingüe entre otros, todos estos documentos están que de una u otra forma dan la consigna, la atención, pero quienes también deben conocerlo, aplicarlo y llevarlo a la práctica son los docentes, entonces podremos decir que la formación docente aun le hace falta la sazón para que recupere la contextualización, diversificación la inclusión de la comunidad a la escuela. 
Las normas y las leyes no nos sirven de mucho que estén aprobados, si no existen suficientes elementos de auto aceptación, auto identitación, auto aprecio a lo propio, hacia la cultura en la que se ha desarrollado como docente, pero sobre todo donde ha pasado una gran parte de su vida en la interacción familiar en esa cultura inmersa donde ha crecido, ya que el docente influye mucho en la formación de los educandos en el aprecio por la cultura, la identidad, identificación de identitación, la inclusión, la aceptación de que forma parte de un grupo, de una comunidad de un pueblo con características propias que lo difieren de otros grupos pero que le pertenecen como un legado de sus ancestros y que ha traido de generación en generación.

El método inductivo intercultural para mayor funcionalidad requiere que como docente tenga una aceptación propia de su originalidad de sus raíces, de su legado. Que se reconozca como parte de un legado, que se le ha heredado, y que ha sido dominado por una cultura distinta occidental, que requiere empoderamiento, dentro de la formación educativa escolar, porque ha sido conquistado en una ideología externa de lo que existe en su entorno, que ha estado inmerso en una ambivalencia de dominación, sumisión. Que ha sido colonizado desde el enfoque escolar, porque se ha preparado para atender a un capitalismo que se ha olvidado de esa relación propia que existen en los grupos originarios, pensamiento-corazón-identitación, que requiere que acepte lo que se le impone a través de un uniforme, de una forma de vestir que esta fuera de su contexto y para poder ser aceptado en esa interacción los alumnos, padres de los pueblos originarios rehúyen, cada vez se ven más personas que olvidan su indumentaria por poner un uniforme a sus descendientes.

Con un suspiro muy profundo le ponen el uniforme escolar, ya que están aceptando que al permitir vestir de esta forma, es por su bien, porque va a ser letrado, porque va a estudiar para ser alguien en la vida, que a pesar de ser ese alguien desde ese momento expone y pone en manos de la escuela esa transformación que no le será para volver o desenvolverse con la visión de su pueblo si no con otra dimensión en la que dudara de que si lo que tiene en su contexto es tan válido como la escuela lo propone, todo debe ser cumplir esta norma para poder ser parte de la institución, tan es así el caso, que como experiencia comento que desde hace 20 años aproximadamente y 15 , donde no se hacía obligatorio portar el uniforme aprobado por el estado, impuesto por los maestros, los niños y las niñas de la comunidades asistían a clases con la indumentaria de la comunidad, portándola con seguridad, orgullo, confianza, tranquilidad, emoción. 
Después de la imposición - sumisión en estos tiempos solicitarle al alumno usar la indumentaria propia de su comunidad, significa, ofensa, imposición, discriminación, exclusión, denigración, vergüenza, porque lo mejor es portar el uniforme que el estado ha regalado e incluso el estado es a quien hay que obedecer en este sentido porque el uniforme es lo mejor, ya que implica ser parte de un estado democrático e inclusivo, sin embargo para el que se resiste dejar de utilizar este elemento con significado propio de su territorio al que pertenece a este espacio significa ser rebelde que no quiere la modernidad, ni la civilización, quiere ser diferente, ¿pero entonces se hace la pregunta ¿Qué es la modernidad? ¿Qué es la civilización’ ¿Por qué permitir y aceptar que nuestras culturas no tienen, ni tenían civilización, ni su propia modernidad? Si nuestros ancestros tenían su propia técnica, su enseñanza-aprendizaje, su pedagogía comunitaria, de las diferentes ramas y asignaturas, a las que el currículo había excluido de manera nacional.

Dentro de cada cultura, comunidad, si existen contenidos, temas, conocimientosaprendizajes que permiten el abono a las asignaturas, ramas, contenidos para el buen vivir, e interactuar con distintas culturas. Pero ahora ¿Cómo está visto el buen vivir desde estos espacios recónditos, enajenantes, diferentes excluidos' el buen vivir no es tener lujos, vanidad, artefactos inútiles, distractores del mundo real? El buen vivir es reconocer que estoy bien, porque tengo salud, no me enfermo, lo que como no afecta mi organismo, mente, cuerpo, vida, lo que obtengo no es para sentirme superior o que hay gente superior e inferior, que es para una anidad innecesaria, que la interacción con el medio no es para dañarla más sino para crear un espacio donde yo soy parte de ella y ella de mí, que si yo la daño, el perjudicado soy yo, porque lo que le doy, e lo devuelve en cantidad sea bueno o malo al final de cuentas en la medida en la que yo cuide a lo demás que está en mi entorno es en esa medida que volverá a mí.

La planeación desde el método implica conocer y reconocer todo lo que hay de manera palpable visible y no visible, de conocimiento empírico en el entorno donde se desenvuelve desde la cultura material e inmaterial, la cultura como sistema de creencias y cosmovisiones, la cultura ecológica, la cultura como medio propio de fomento al lenguaje florido en la lengua ordinaria, propia de la comunidad. Considerando el calendario socio natural instrumento que guarda, registra y apertura todo lo que hay en el entorno, territorio. El seguimiento a las actividades que realizan tanto como los comuneros, los niños, el comportamiento vegetal, el comportamiento animal, el comportamiento climático, temporadas, son un pretexto oportuno de partida, la búsqueda de pretexto al que hace alusión María Bertely Busquets en el taller regional 
interinstitucional. Está en el calendario. Entonces hablar de planeación desde el método en vinculación con los programas escolares es establecer el hilvanado que la indumentaria de los pueblos originarios tiene pero para poder realizarlo se necesita conocerlo, sabe ¿cómo? se ha hilvanado ¿cómo están entrelazados los conocimientos? Darle sentido, color, atracción, e imaginación, conocimiento, enseñanza-aprendizaje, para que de ello se pueda derivar el tema, el contenido, el aprendizaje esperado que permita, no solo atender a un currículo escolar priorizado externo, sino que permita su auto reconocimiento.

Cuando se habla de reconocimiento es que debido a las movilizaciones existentes de saberes en las comunidades predominantes son los que los libros de texto contienen o el currículo escolar en su defecto marca lo que se puede encontrar en el internet, en el google, es la que empodera a pesar de que en un espacio reducido de la misma comunidad los que se han resistido a olvidar esos saberes que son propios de todos lo mantienen, solo que en sus excepciones está quedando encuadrado ya solo en los mayores de 40 años y los menores a pesar de estar inmersos en la misma comunidad saben que se hace dentro de su comunidad, sabe que en algún momento al interior de su familia si se ha practicado, reconoce que es parte de su comunidad, pero se niega aceptar que puede ser el hilo que pueda establecer ese vínculo de unión de aprendizajes entre lo propio y lo externo pero que permita mantener conocimiento como parte de su identidad de su cultura pero, que a su vez también aprenda el empírico comprobado por su civilización le permitan verificar sus hipótesis desde una óptica científica conforme a lo que la ciencia lo requiere.

Para poder lograr el auto reconocimiento de saberes de la comunidad es necesario que los educandos establezcan lazos de comunicación desde los más pequeños hasta los más adultos y que la escuela sea un espacio abierto de los mayores, para que compartan sus saberes con las nuevas generaciones, que a su vez se requiere que el docente también acepte que hay muchos conocimientos generados desde ese entorno, territorio, dentro de la comunidad que también los desconoce, que existen saberes que se han guardado celosamente por el miedo a la discriminación, la negación de ser benéfica a la comunidad, porque para los ojos de los externos, es maléfico e incitador de energías malas, dañinas, a la vista de todos, los demás casados con las religiones, entonces se está hablando de que el maestro es quien va a recuperar conocimientos a través de distintas técnicas de investigaciones, que va a fungir como un andamio, e investigador que propiciara que sus alumnos también sientan la necesidad por querer conocer más de lo que 
hay en su entorno, de lo que sus ancestros guardan celosamente pero que es necesario hacerlo visible para que su técnica, conocimiento no se desaparezca.

Dentro de las distintas técnicas de investigación que existen, las prioritarias por decirlo de esta forma son historias de vida, técnicas de observación participante, porque en ellas existen mayores elementos de acercamiento, aceptación por parte de los comuneros en el entendido que en las historias de vida se establece una comunicación en donde permea la confianza, seguridad, aceptación, e las comunidades arraigadas una vez que el docente lo sienten parte de la familia le facilitan la información, la comunidad también observa, si existe identitación, hablar de identitación es sentir desde el corazón para facilitar todo o una parte, cuando se habla de todo es que con datos precisos, informan, cuestión de disponibilidad de tiempo y capacidad de memorización y registro para guardar la información, comparten técnicas, recursos que han utilizado, sus principales recursos, el fin social de las actividades, también se comparten historias personales enfocadas con el territorio, y cuando se hace mención que es una parte es que solo se comparten algunos datos, cerrándose a que el recuerdo no está presente, o que no se tienen más datos.

El método inductivo intercultural, recupera también la teoría de la actividad, en donde está inmersa la formación comunitaria, religiosa, escolar y de convivencia. Cuando se habla de la religiosa no se refiere únicamente a las ideologías de sincretismo impuestas, sino a esa cosmogonía que tienen los pueblos originarios, donde la participación activa en las actividades, es la mejor forma de aprender. Se aprende haciendo, entonces se puede decir que la predominancia es de la ideología de los pueblos, de la comunidad donde se labora, antes que hacerlo por el santo, por la iglesia que predomina, por lo que el sacerdote o la biblia dice, es donde hay una explicación que parte de la interacción hombre-naturaleza, donde no se dice: hay que hacerlo por el bien único para un beneficiado, donde impera el juego de un ganador exclusivo, de un sometido, de un sumiso, o el que tiene mayor autoridad, sino de él, ella y el yo es cambiado por el nosotros, de que hay que hacerlo porque todos o los involucrados seamos beneficiados, si ellos están bien, yo estoy bien, pero ¿Quién es ella? ¿Quiénes son ellos?

La madre tierra es ella a la que hacen alusión, donde está la madre tierra primero, aun en tiempos de crisis, aunque en estos tiempos de modernidad la tierra aun en los pueblos originarios también ya sufre el desplazamiento pero en esta ambivalencia de sobrevivencia, resistencia, sentido cosmogónico y sistema de creencias y cosmovisiones, impera la formación del legado por 
herencia, donde al final en el pensamiento y corazón se decide: "si tengo que hacerlo porque si no se hace aunque yo me acabe, mi caminar se termine vuelvo a la madre tierra y la madre tierra me cobrara mi paso por esta" y no haber hecho algo aunque no palpable para los demás, de que mi pueblo tiene conocimiento por hacerlo, no me ira tan bien, entra en un dilema mental moral por lo que al final termina haciendo lo que el legado cultural le ha heredado ya que la conciencia de que me fue dicho, me fue enseñado, me fue mostrado, lo viví, experimente, observe, fui parte, ajora me toca hacerlo, porque eso es lo que me enseñaron mis ancestros, mis padres y mi obligación como parte de esa herencia inmaterial cultural. Es hacerlo, aunque los demás me miren como raro, como fuera de tiempo, de espacio, esta resistencia de pérdida del legado al que María Bertely hacía hincapié en sus diálogos en los talleres, se empodera al haber un conocimiento disperso, que al justarse toma el poder, o se hace visible ante toda una comunidad pero debe haber algo así como un eslabón que propicie la unión, el hilvanado, la costura, los eslabones son los expertos de esa actividad.

Nuevamente retomo entonces la planeación ¿Dónde quedo? ni más ni menos que en este "enlace de la comunidad y la escuela", donde: "la escuela se apertura a la comunidad" María Bertely en taller ciudad de México, casa Xitla, "cuando María decía que la escuela se apertura a la comunidad desde este entender no es que la escuela propone el contenido desde el programa curricular, sino que el tema parte de la comunidad donde el contenido, el tema son los pretextos para recuperar conocimientos y introducirlos en la escuela y el niño los reconozca, el padre de familia asuma la importancia de entender su propia historia, para generar conocimientos, que al introducir el contenido comunitario a la escuela, la escuela establece así como una devolución de lo que le ha sido arrancada, pero ¿se lograra en un ciclo escolar? Obviamente que no, se requiere de una aceptación muy marcada, de que los mismo padres son lo que también deben asumir que lo propio tiene sus propios contenidos que permiten generar otros, para que también sean los partícipes en la comunidad sean aprendices constantes. Desaprender lo que la escuela con enfoque occidental nos ha enseñado cuesta. Es un trayecto un poco largo, pero que uno acepte y entienda el enlace facilita la participación de los demás.

La planeación es una herramienta fundamental para el desarrollo del trabajo de cada docente, en el método inductivo intercultural no está exento de la elaboración de este material prescindible, ya que en ella se enmarcan la previsión de objetivos, propósitos, recursos, sistematización de actividades contextuales a partir de una frase generadora que considera los 4 
ejes, que son territorio, recurso, técnica y fin social, además de otros elementos que permiten atender en primer momento la valoración del entorno, el reconocimiento de lo propio, de lo que forma parte de una cultura material e inmaterial, de un patrimonio fenomenológico, que a su vez repercute en una identidad comunitaria para posteriormente satisfacer el currículo oficial como documento base articulador.

En la planeación se puede decir que tiene momentos, en la que podemos encontrar la motivación, que parte de una actividad introductoria hacia el contenido en particular, la recuperación de conocimientos previos, actividad social, explicitación, articulación, ampliación y sistematización, para cerrar con un seguimiento y/o devolución del conocimiento a la comunidad, así también en cada momento se consideran acciones secuenciadas, que dentro de las comunidades originarias están implícitas dentro de una actividad, es decir que una actividad como generadora tiene otras actividades dentro de ella que permite la organización, puesta en práctica de diversos conocimientos implícitos en donde se integran de manera articulada los niños, padres, abuelos como participantes y generadores de los conocimientos propios de la comunidad, que se han mantenido a través de un legado cultural oral de generación en generación, la planeación con este enfoque podría decirse que cumple la expectativa de un proyecto.

La planeación también considera las 4 dimensiones que son: Ontológico, Axiológico, Pedagógico y Político, que se recupera, con los 4 ejes en vinculación. Donde: Una de las características importantes del método es conservar la cultura en su generalidad, la cosmovisión y sistemas de creencias de los pueblos indígenas considerándolo como: el punto de partida, de retroalimentación constante, para revalorar, fortalecer, aprovechar éste conocimiento originario que hace una invitación constante al buen vivir, visto como una vida saludable, respetable, integrada, no como un espacio de comodidad, presunción, lujo y económico, sin embargo no está libre de las tentaciones que la modernidad trae, del bombardeo publicitario consumista, a la que nuestros pueblos originarios se ven expuestos continuamente.

Para recuperar todos estos elementos visibles en la planeación conforme el método inductivo intercultural se ha propuesto, la organización de trabajo a través de milpas educativas, que prácticamente llevan este enfoque, con la finalidad de que siempre se retomen actividades de la comunidad ya que una milpa no únicamente se enfoca a la siembra de maíz, sino como su nombre lo dice: proviene de la toponimia náhuatl, donde: mili de miloua- siembra, ipa sobre de, y 
la siembra vista desde las comunidades no únicamente están enfocadas a un solo cultivo, los originarios son policultivos donde existe la convivencia del maíz, con la calabaza, frijol, flores, otro tipo de hierbas, una siembra de un producto con mayor predominancia pero que existe la convivencia con otros dentro del mismo espacio, que no únicamente se siembra un solo producto exclusivo limitando el crecimiento de otros, sino que también considerando la necesidad, la importancia y influencia. La interacción, ya que permiten el desarrollo y la combinación de nutrientes para que tenga un sabor delicioso a la hora de preparar la comida, además de que esa interacción en la siembra proporciona salud, entonces se puede decir que la milpa educativa no es comparado con un huerto escolar, huerto familiar, huerto comunitario, práctica social, actividad.

¿Por qué no se compara con estos? La milpa educativa es salir de la escuela, ir al territorio donde existe la siembra, pero esa siembra como su nombre lo dice educativa, no únicamente es vista desde el enfoque de lo que produce la tierra, sino también lo que produce la mente, el cuerpo, la vida - la muerte, la interacción de la humanidad con la naturaleza, para una educación, cuando se habla de esto es porque, la educación está en todos los espacios donde se hacen actividades, que muchas de las veces no son única actividad son poli actividad, que van de la vida y la muerte, a la interacción hombre naturaleza, porque por ejemplo cuando hay una fiesta patronal aunque es solo una fiesta existen varias actividades al mismo tiempo que se van realizando en un espacio que existen expertos, aprendices, que todos colaboran, hombres, mujeres, niños, acordes a una división de trabajos, que cada uno puede hacer con mayor habilidad. Por lo tanto, esto permite la contextualización y diversificación de actividades ya que el que no le gusta hacer flores, pero sabe rajar leña, decide donde quiere estar, ¿Qué es lo que quiere hacer?

Por lo tanto al permitir este involucramiento de actividades en la milpa educativa no solo se limita a una actividad si no a lo que hay en el entorno además de que se busca el empoderamiento, la aplicación de los acuerdos nacionales e internacionales en relación a la atención de los pueblos originarios, que a través de ellos se aprende a leer, escribir, se generan matemáticas, se reconoce la formación cívica y ética, exploración y conocimiento del mundo natural y social, convivencia, solidaridad entre otros.

Para cerrar se hace la presentación de un formato que no puede ser encuadrado en su totalidad, también puede ser descriptivo, pero que permite entender un poco más allá de la planeación.

Dossiê Práticas de bem viver: diálogos possíveis entre o Núcleo Takinahakỹ e Milpas Educativas 
Cuadro - Planeación.

\begin{tabular}{|c|c|c|c|c|c|}
\hline $\begin{array}{l}\text { Secuencia } \\
\text { didáctica }\end{array}$ & $\begin{array}{l}\text { Acciones, } \\
\text { Espacios y } \\
\text { tiempos }\end{array}$ & $\begin{array}{l}\text { Participantes } \\
\text { y recursos }\end{array}$ & $\begin{array}{l}\text { Conocimientos } \\
\text { propios, } \\
\text { significados }\end{array}$ & $\begin{array}{l}\text { Conocimientos } \\
\text { escolares } \\
\text { universales }\end{array}$ & $\begin{array}{l}\text { Evaluación, } \\
\text { ¿que, quienes, } \\
\text { cuando y como } \\
\text { se evalúa? }\end{array}$ \\
\hline $\begin{array}{l}\text { 1.- Motivación y } \\
\text { conocimiento } \\
\text { previo }\end{array}$ & $\begin{array}{l}\text { Dentro de los } \\
4 \text { momentos } \\
\text { se consideran }\end{array}$ & & $\begin{array}{l}\text { En los } 4 \text { momentos } \\
\text { se recuperan los } \\
\text { conocimientos }\end{array}$ & $\begin{array}{l}\text { Estos } \\
\text { conocimientos } \\
\text { se retoman del }\end{array}$ & $\begin{array}{lr}\text { El } & \text { docente } \\
\text { decide } & \text { ¿Cómo } \\
\text { valorar } & \text { cada }\end{array}$ \\
\hline $\begin{array}{l}\text { 2. Actividad social } \\
\text { (organización y } \\
\text { recomendaciones }\end{array}$ & $\begin{array}{l}\text { los: } \\
4 \text { ejes y } \\
4 \text { dimensiones }\end{array}$ & & $\begin{array}{l}\text { propios de la } \\
\text { comunidad en su: } \\
4 \text { ejes } \\
4 \text { dimensiones }\end{array}$ & $\begin{array}{l}\text { programa en } \\
\text { vinculación con } \\
\text { los } \\
\text { conocimientos }\end{array}$ & $\begin{array}{l}\text { 'momento? } \\
\text { Aunque en } \\
\text { cuestión de las } \\
\text { actividades que }\end{array}$ \\
\hline $\begin{array}{l}\text { 3.- Explicitación } \\
\text { articulación, } \\
\text { ampliación y } \\
\text { sistematización }\end{array}$ & & & & orle & $\begin{array}{l}\text { realiza la } \\
\text { comunidad } \\
\text { tienen su propia } \\
\text { forma de } \\
\text { valoración. }\end{array}$ \\
\hline $\begin{array}{l}\text { 4.- Seguimiento o } \\
\text { devolución }\end{array}$ & \multicolumn{3}{|c|}{$\begin{array}{l}\text { En este momento, es que con la comunidad en } \\
\text { general se comparte lo que se aprendió al realizar la } \\
\text { actividad. Para que todos lo aprendan. }\end{array}$} & & \\
\hline Observaciones & & & & & \\
\hline
\end{tabular}

Fonte: Georgina Marcelo Hernández. 


\section{Referência}

BERTELY, Maria Busquets. SARTORELLO, Stefano. Milpas Educativas: Laboratórios socionaturales vivos para el Buen Vivir. Universidad Iberoamericana, 2017.

Submetido em 2 de julho de 2019.

Aceito em 05 de agosto de 2019.

Publicado em 8 de agosto de 2019. 